\title{
Risk of Femoral Bone Fractures in Hip Arthroplasties during Sideway Falls
}

\author{
Abdul Halim Abdullah, Mitsugu Todo, Yasuharu Nakashima, and Yukihide Iwamoto
}

\begin{abstract}
Sideway falls are potential accident to elderly population and very risky for hip fractures. The tragedy may become worst for patients with hip arthroplasties. Sudden impact loading that occurs during fall may lead to femoral fractures and joint failures. Different techniques of falling give different consequences and reflect the long term stability. In this study, risk of bone fractures was predicted during sideway falls using finite element analysis with damage formation criterion. Inhomogeneous model of femur was developed from CT-based data for computational analysis. Femur models with resurfacing hip arthroplasty (RHA) and total hip arthroplasty (THA) were constructed to estimate the fracture possibility in hip arthroplasties. Four different configurations of sideway falls were considered namely FC1, FC2, FC3 and FC4 which presenting different hip loading direction and boundary conditions. Hip impact forces were normalized to body weight (BW) and varies from 1BW to 5BW were applied to each configuration. The results showed that the patterns of bone fractures were different between intact, RHA and THA femurs. Numbers of element failures were highest in RHA femur while lowest in THA femur for almost all configurations. Fractures locations were predicted at trochanteric region due to boundary condition during fall. Indication of fractures inside the canal shaft of THA femur due to bending effects may contribute to prosthesis loosening and instability.
\end{abstract}

Index Terms-Hip resurfacing, total hip arthroplasty, sideway falls, bone fractures, damage formation.

\section{INTRODUCTION}

Total hip arthroplasy (THA) and Resurfacing hip arthroplasty (RHA) are two common approaches for hip osteoarthritis treatment. Issues of long term stability of implant and bone growth are part of the debate in promoting both procedures. Biomechanical factors such as bone absorbtion, bone fractures and prosthesis loosening are to be considered for primary and long term successful. Also, clinical and patient-reported outcomes have to be taken into account, including return to function, pain relief and increase mobility.

Computational biomechanical analysis had widely implemented to promote the primary and long term prediction. Different loading behavior for daily activities such as walking and stair climbing were demonstrated in

Manuscript received May 16, 2014; revised July 16, 2014

A. H. Abdullah is with the Interdisciplinary Graduate School of Engineering Sciences, Kyushu University, Kasuga 816-8580 Japan, on study leave from Faculty of Mechanical Engineering, Universiti Teknologi MARA Selangor, Malaysia (e-mail: abdul.halim.abdullah.822@s.kyushu-u.ac.jp).

M. Todo is with Research Institute for Applied Mechanics, Kyushu University, Kasuga 816-8580 Japan (e-mail: todo@riam.kyushu-u.ac.jp).

Y. Nakashima and Y. Iwamoto are with the Department of Orthopaedic Surgery, Kyushu University, Fukuoka 812-8582 Japan. predicting stress behavior, potential of failure and implant stability [1]. In addition, patients with hip osteoarthritis and hip arthroplasties were also at risk of bone fractures especially to elderly. Instead of having weaker bone, they are more likely to fall due to imbalance, medication side effects and difficulty avoiding environmental hazards [2]. The external loading created during sideway falls will initiate to sudden and high impact loading to the hip contact. Risks of falling for hip arthroplasties patients are higher due to gait adaptation and instability [3].

The purpose of the current study were 1) to develop inhomogeneous 3D model of intact femur and femur with arthroplasties (THA and RHA) based on CT-based data and 2) to examine the bone fractures mechanism of intact, RHA and THA femurs under different loading configuration of sideway falls.

\section{MATERIALS AND METHOD}

\section{A. Development of Inhomogeneous Bone Model}

A 3D femur model was constructed from computed tomography (CT) based images of 54-years old male. The models were designed using tetrahedral solid element with a size of $3 \mathrm{~mm}$ for the whole bone. The mechanical properties of the bone element were computed by Hounsfield unit value to determine inhomogeneous model. Young modulus and yield stress of each element were calculated based on Keyak et al. [4] while Poisson ratio for each element is set as 0.4. Distribution of young modulus in the inhomogeneous model was illustrated in Fig. 1.

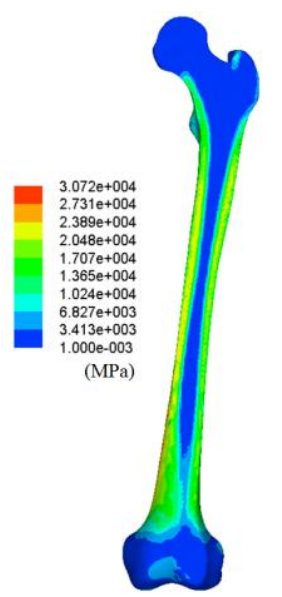

Fig. 1. Distribution of young modulus in inhomogeneous femur model

\section{B. Material Properties}

CAD data of hip resurfacing and total hip arthroplasty were imported and implanted into femoral bone and 
presented as RHA and THA femur models, respectively. The femoral head was resurfaced and implanted with arthroplasty in RHA while the femoral was cut off in THA femur, as illustrated in Fig. 2. Prosthesis stem of THA was modeled as Ti-6Al-4V material while femoral ball as Alumina properties. Meanwhile, RHA implant was assigned as Co-Cr-Mo material. Details of material properties for each component were summarized in Table I [4]. Contact between both implants and bone were considered to be perfectly bonded at the interface.

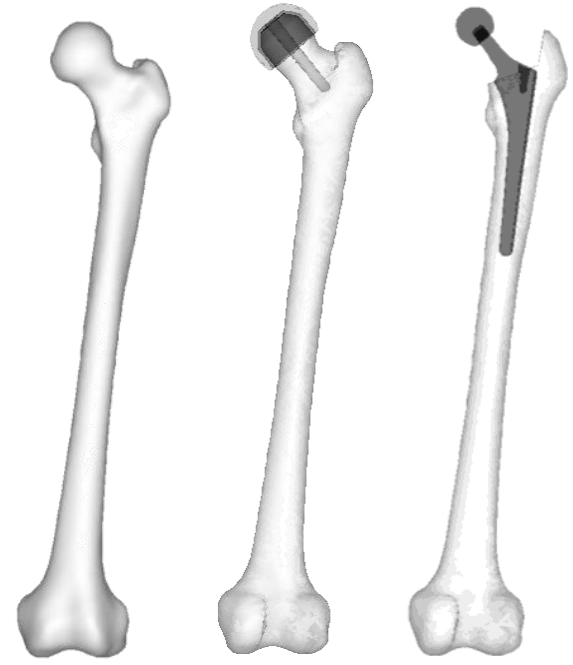

Fig. 2. 3-dimensional models of intact, RHA and THA femurs.

TABLE I: MATERIAL PROPERTIES OF HIP ARTHROPLASTIES

\begin{tabular}{lccc}
\hline \hline Properties & Ti6Al4V & Alumina & Co-Cr \\
\hline Elastic Modulus (GPa) & 114 & 370 & 230 \\
Poisson Ratio & 0.34 & 0.22 & 0.30 \\
Critical Stress (GPa) & 0.88 & 0.40 & 0.94 \\
Yield Stress (GPa & 0.97 & 3.00 & 2.70 \\
Density (g/cm3) & 4.43 & 3.96 & 8.28 \\
\hline \hline
\end{tabular}

\section{Loading and Boundary Conditions}

Four different types of loading and boundary conditions were assigned to demonstrate different sideway falls [5]. Each configuration exhibits different loading directions at angle $\alpha$ (with reference to the long axis of femur in frontal plane) and $\beta$ (with reference to femoral neck axis in horizontal plane) as proposed in Bessho et al. [5]. Fig. 3 and Table II described the different angle $\alpha$ and $\beta$ for each configuration. The variety of falling configurations (FC) were labeled as FC1, FC2, FC3 and FC4 and illustrated in Fig. 4.

Experimental study to measure the impact forces at the hip during sideway falls was previously conducted by Groen et al [6]. The study concluded that proper fall techniques (eg. Martial arts) will reduce impact velocity and loading. Hip impact force (normalized to body weight, BW) may reduce from $3.9 \mathrm{BW}$ to about $2.8 \mathrm{BW}$. In this study, different loading magnitudes were assigned for each configuration to predict sudden impact of falls. Increments of loading from 1BW to $5 \mathrm{BW}$ were considered to predict the fracture patterns and locations during falls with different configurations. Finite element analyses combined with a damage mechanics model were performed to predict bone fractures in both arthroplasty models and intact femur.

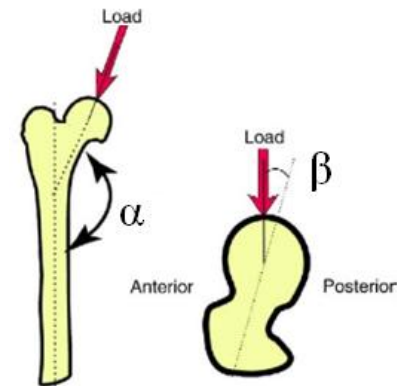

Fig. 3. Definition of different loading direction $\alpha$ and $\beta$ at coronal (left) and axial (right) views.

TABLE II: DESCRIPTION OF DIFFERENT ANGLE DEGREE

\begin{tabular}{ccc}
\hline Configuration & $\alpha$ & $\beta$ \\
\hline FC1 & 120 & 0 \\
FC2 & 60 & 0 \\
FC3 & 60 & 15 \\
FC4 & 60 & 45 \\
\hline
\end{tabular}

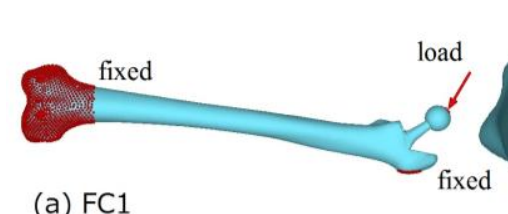

(a) FC1

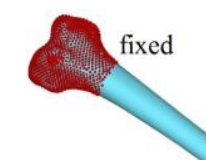

(b) FC2

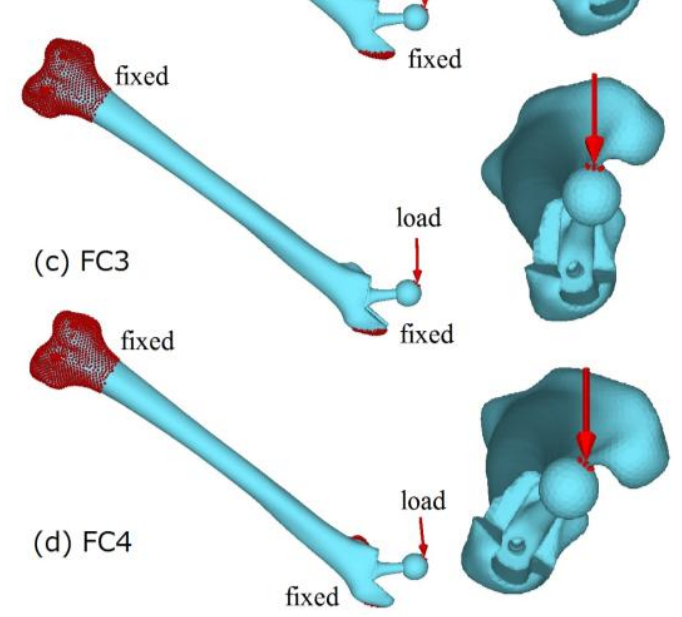

Fig. 4. Loading and boundary conditions for different falling configurations.

\section{RESUlTS AND DISCUSSION}

Risk of femoral fracture in all femurs was predicted computationally using commercial biomedical software, Mechanical Finder, v6.1 in corresponding to damage formation criterion. Fracture mechanism of femurs was calculated based on prediction by Keyak et al. [7]. The load bearing strain was set to 3000 micron which representing bone loading that leads to bone formation. The test tensile strength was set to $80 \%$ of the yield strength determined from the CT images [8], [9].

\section{A. Changes in the Distribution and Numbers of Element Failure}

Increments of failure elements by increasing of body 
weight (BW) loading were presented in Fig. 5 for different configurations. Total of elements failure were accumulated for both tensile and compressive direction. Patterns of changes for failure element were increased exponentially for all configuration and femur models. Configuration FC1 showed less numbers of element failures as compare to other configurations which range up to only 1700 elements at 5BW while others range to 7000 of fracture elements.

Configuration $\mathrm{FC} 1$ indicates that risk of bone fractures are high in THA femur followed by RHA and intact femurs. But, the intact femur shows higher number of element failures

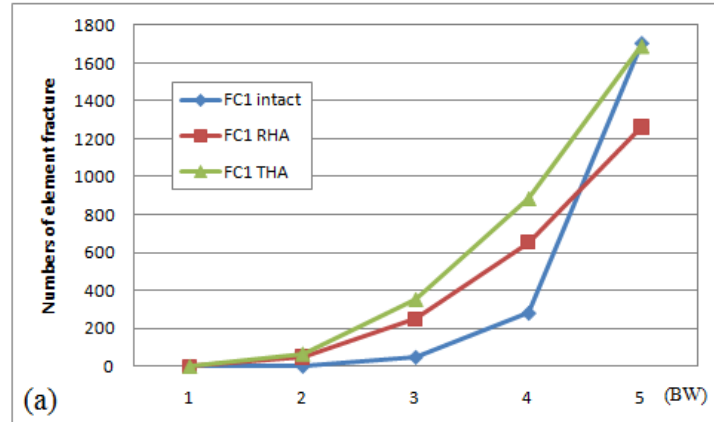

(a)

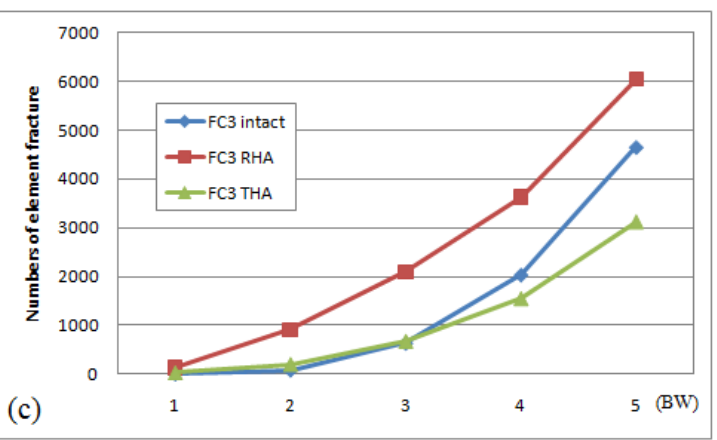

when the loading exceed 5BW as projected in Fig. 5(a). Differs to other configurations, fractures or failure element of bone were expected to be dominant in RHA femurs while minimum in THA femurs. The patterns were almost comparable between configuration FC2, FC3 and FC4 along the increasing body weight loading as shown in Fig. 5(b), 5(c) and 5(d), respectively. Nevertheless, the elements of failures are expected to be peak up to 8000 element in intact femur at $5 \mathrm{BW}$ in configuration FC4 (Fig. 5d). The incidence may be occurred due to collateral damage of the femoral bones caused by the high impact.
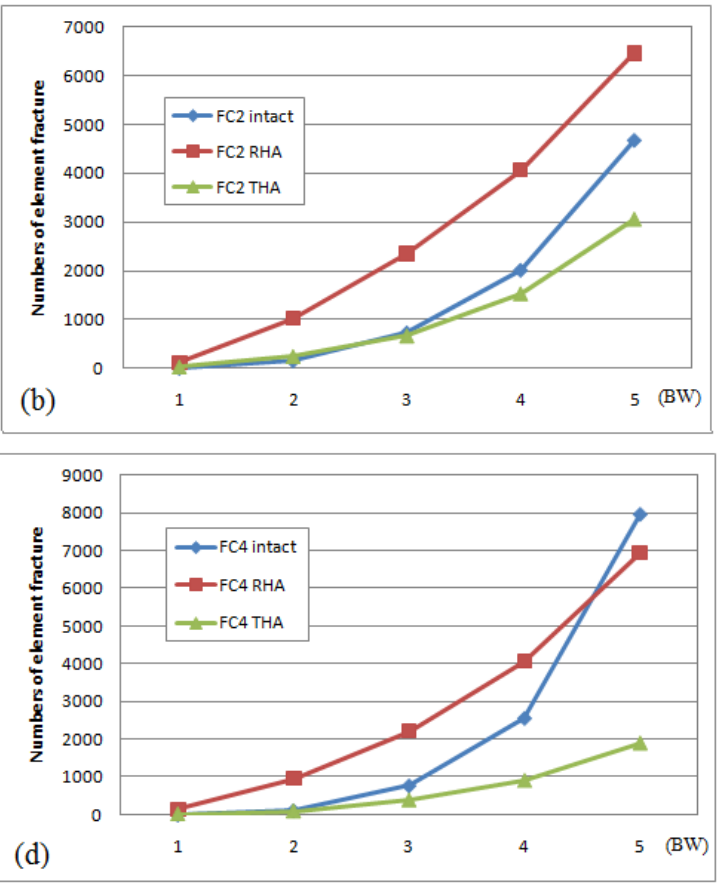

Fig. 5. Changes of element failures by increasing of body weight (BW) loading for different falling configurations (a) FC1, (b) FC2, (c) FC3 and (d) FC4 between intact, RHA and THA femurs.

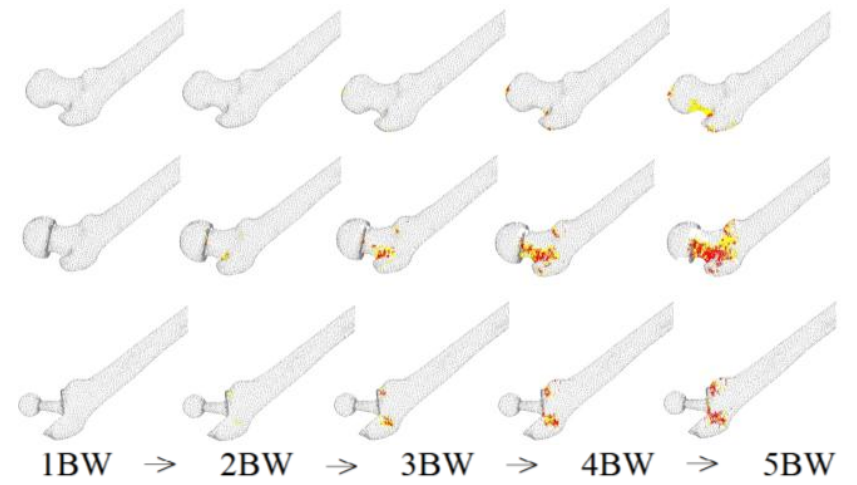

Fig. 6. Patterns of bone fractures in intact, RHA and THA femurs in increasing body weight from $1 \mathrm{BW}$ to $5 \mathrm{BW}$ loading (left to right) for configuration FC4.

Changes of element failure distribution in increased body weight loading contribute to the pattern of bone fractures in intact, RHA and THA femurs. Fig. 6 illustrated the changes in the distributions of the elements failure for configuration FC4 for all femur models at posterior-anterior (P-A) view. Tensile fractures were indicated in red while compression fractures in yellow. No sign of fractures were expected at lower impact loading (below 1BW). The failures of elements were expected to initiate at $2 \mathrm{BW}$ and $3 \mathrm{BW}$ for RHA and THA models, respectively. While fractures of the intact femur were clearly indicated at 5BW and dominated at the upper neck of femoral.

Fractures of RHA femur were expected to occur at femoral neck when the load reached 3BW. In higher load at 5BW, the additional fractures location was pointed out along the inter-trochanteric crest region. For THA femur model, the bone fractures were projected to locate at proximal medial and lateral region of femoral cutting. The direction of hip loading during fall may lead to lateral-medial bending effects to the prosthesis stem and replicate force to the proximal cut of the femur. Fall area at lesser trochanter region may also contribute to stress concentration and continue to fail.

\section{B. Prediction of Bone Fracture Location in Intact, RHA and THA Femurs}

Damage formation of bone fractures occurred in all configurations with different locations. Fig. 7 illustrated the different predicted locations of bone fractures for configurations FC1-FC4 at 5BW loading. In general, loading direction and boundary condition which differentiate the falling configurations lead to the fracture locations. FC1 and FC2 configurations defined similar loading angle $\left(0^{\circ}\right)$ of $\beta$ but contrast in $\alpha\left(120^{\circ}\right.$ and $\left.60^{\circ}\right)$. Different $\beta$ angle of loading direction were applied in FC2, FC3 and FC4 configurations at $0^{\circ}, 15^{\circ}$ and $45^{\circ}$, respectively.

Bone fractures occurred at the area of impact loading and boundary conditions due to high stress concentrations. In 
addition, direct impact hip loading to the femoral head of intact femur give high stress concentration. Fractures in tensile direction were designated in red element while yellow element shows compression failures. At 5BW of hip loading, fractures were projected to occur in all falling configurations and all femur models.

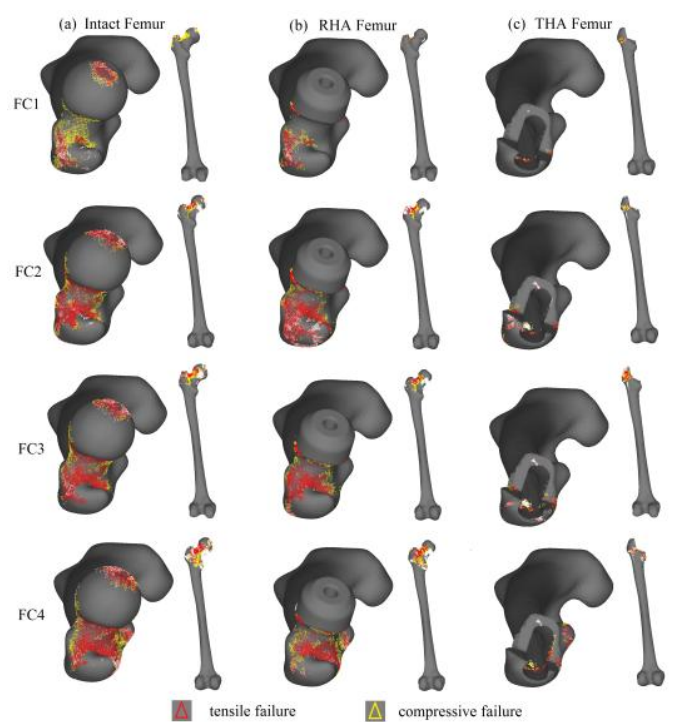

Fig. 7. Prediction of femoral fracture locations for intact, RHA and THA femurs at 5BW loading with different views, top (left) and P-A (right).

For intact femurs, bone fractures were located at upper femoral neck regions in every configuration. Risk of femoral damage at femoral neck was higher in FC4 configuration with additional predicted fractures location at bottom neck and inter-trochanteric crest region, as shown in Fig. 7(a). Fractures in RHA femurs signified almost similar pattern to that predicted in intact femur except for FC1 configuration. Region of upper femoral neck experienced tensile failure while bottom neck with compressive failure. Pin shaft of resurface arthroplasty which implanted into the femoral ball may reflect to axial and bending load to the femoral head. Furthermore, bending load that occurred due to hip loading angles contributed to the stress concentrations and failure outcomes.

Pattern of femoral fractures in THA femurs shows different trend compared to intact and RHA femurs. Presence of prosthesis stem inside the canal creates mismatch material to the femoral shaft. Phenomenon of stress shielding and bone remodeling were widely discussed in promoting long-term performance. The predicted fractures were initiated at the proximal canal femur. The areas were believed to represent low modulus of elasticity or known as cancellous bone. The hip loading applied had induced bending moment to the prosthesis stem.

Consequently, the proximal resection area of the femur was reflected and stresses were dominated. Higher stress concentrations at low elastic modulus material were tended to fracture. Damage formation that occurred inside the proximal canal femurs may lead to prosthesis loosening and decrease implant stability. Bone fractures also projected at the greater trochanteric region for all configurations at the higher hip loading $(5 \mathrm{BW})$.

\section{CONCLUSION}

The inhomogeneous FE models of femurs are well developed in this study to predict bone fractures for intact, RHA and THA femurs during sideway falls. Different locations of fracture are predicted in each configuration due to different loading direction and boundary conditions. Predicted fractures for intact and RHA femurs are dominant at femoral neck region in all configurations. While, trochanteric region and proximal canal for THA femurs and expected to lead for stem loosening and instability.

\section{REFERENCES}

[1] A. D. Speirs, M. O. Heller, W. R. Taylor, G. N. Duda, and C. Perka, "Influence of changes in stem positioning on femoral loading after THR using a short-stemmed hip implant," Clinical Biomechanics, vol. 22, 2007, pp. 431-439.

[2] L. C. Brunner, L. Eshilian-Oates, and T. Y. Kuo, "Hip fractures in adults," Am Fam Physician, vol. 67-63, 2003, p. 537.

[3] M. L. Beaulieu, M. Lamontagne, and P. E. Beaule, "Lower limb biomechanics during gait do not return to normal following total hip arthroplasty," Gait and Posture, vol. 32, 2010, pp. 269-273.

[4] J. H. Keyak, H. B. Skinner, J. A. Fleming, "Effect of force directionon femoral fracture load for two types of loading conditions," J Orthop Res., vol. 19, 2001, pp. 539-544.

[5] M. Bessho, I. Ohnishi et al., "Prediction of proximal femur strength using a CT-based nonlinear finite element method: Differences in predicted fracture load and site with changing load and boundary conditions," Bone, vol. 45, 2009, pp. 226-231.

[6] B. E. Groen, V. Weerdesteyn, and J. Duysens, "Martial arts fall techniques decrease the impact forces at the hip during sideways falling," J Biomech, vol. 40, 2007, pp. 458-462.

[7] J. H. Keyak, S. A. Rossi, K. A. Jones, H. B. Skinner, "Prediction of femoral fracture load using automated finite element modeling," $J$ Biomech, vol. 31, 1998, pp. 125-133.

[8] T. S. Kaneko, M. R. Pejcic, J. Tehranzadeh, and J. H. Keyak, "Relationships between material properties and CT scan data of cortical bone with and without metastatic lesions," Med Eng \& Phy, vol. 25, 2003, pp. 445-454.

[9] D.Taylor, J. G. Hazenberg, and T. C. Lee, "The cellular tranducer in damage-stimulated bone remodeling: a theoretical investigation using fracture mechanics," J Theo Bio, vol. 225, 2003, pp. 65-75.

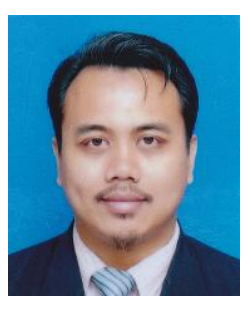

Abdul Halim Abdullah obtained his bachelor of engineering (mechanical) from Universiti Sains Malaysia and master of engineering in biomechanical engineering from Universiti Teknologi Malaysia. Currently he is a PhD student in Kyushu University, Japan. He is a senior lecturer at Faculty of Mechanical Engineering, Universiti Teknologi MARA, Malaysia and involved in computational biomechanics research.

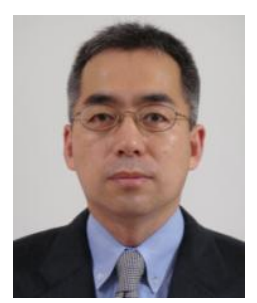

Mitsugu Todo obtained his bachelor and master's degrees of engineering from Kyushu University, Japan, and PhD from the Ohio State University, the United States of America. He is currently an associate professor of the Research Institute for Applied Mechanics, Kyushu University, and is working on biomaterials for osteochondral tissue engineering and biomechanics of orthopedic implants. 